\title{
Preparing a paper for publication: an action plan for rapid composition and completion
}

\author{
Jeffrey A. Harvey
}

Netherlands Institute of Ecology (NIOO-KNAW), Centre for Terrestrial Ecology, P.O. Box 40, NL-6666 ZG Heteren, The Netherlands (e-mail: j.harvey@nioo.knaw.nl)

Received 18 Feb. 2008, revised version received 19 Aug. 2008, accepted 22 Aug. 2008

Harvey, J. A. 2009: Preparing a paper for publication: an action plan for rapid composition and completion. - Ann. Zool. Fennici 46: 158-164.

An important challenge for scientists, especially those early in their careers, is preparing an effective article for submission to a peer-reviewed journal. Here, I present a number of suggestions on how it could be accomplished. This action plan addresses (1) how to approach a topic by developing a story line connected with what is already known in the field, (2) how to most efficiently organize and sequence one's efforts by starting with the descriptive parts of the manuscript and subsequently moving to the more interpretive parts, and (3) the advantages of using bibliographic software to facilitate quick and accurate referencing. I suggest that authors should aim to produce a story that does not overcomplicate the topic under investigation while at the same time presenting a full and accurate coverage and interpretation of the data. Importantly, the preparation of manuscripts becomes easier with time and practice, as individuals hone their own style and approach to this task.

\section{Introduction}

The ability to formulate hypotheses, to independently conduct experiments, and to generate empirical data are important pre-requisites for a successful scientific career. Moreover, it is equally vital to be able to correctly analyze the data and to write up the results for publication in peer-reviewed journals. The latter requirement is a stumbling block for many inexperienced writers, particularly those for whom English is not their first language. At the same time, many native English speakers (or those fluent in the language) also often find it most difficult to be able to write up the results of their research. Given that science is often cruelly referred to a 'publish-or-perish' profession, 'writers block' can thus prove to be a major impediment in the building of a career in science.

Clearly, different individuals have their own ways of approaching the task of writing up the results of their empirical research. As a young scientist becomes more experienced and their publication record grows, they generally find that preparing manuscripts for peer-review becomes a steadily easier task. Some (including myself) may even change the way they approach the problem. For instance, early during my scientific career, I found it necessary to write the entire manuscript initially by pen in a paper notebook. There was no particular order to what I wrote, but instead I just put ideas onto paper as they came into my head. This was because my ideas often flowed much more quickly than my ability 
to enter them coherently into the primitive text processing software I had at the time, whereas I could scribble them onto paper much more effectively. Furthermore, ideas jotted onto paper could be more easily changed, altered or deleted. However, with time this habit began to fade and I learned to put my ideas straight into a digital file. Modern technology certainly helped, but there were other factors involved as well, including a growing ability to jot ideas onto a computer page rapidly and efficiently. When this happened, the notepad was usually abandoned.

The bottom line is that in 'writing up' everyone will adopt a style that suits them personally. In spite of this, a short guide for preparing and writing up manuscripts effectively can still be helpful for many young researchers with limited experience in this area. This short paper is therefore aimed at understanding ways to most effectively tackle the problem of preparing manuscripts. Several excellent books have been published over the past 15 years (e.g. Williams 1990, Booth 1993, Gustavii 2003, Malmfors et al. 2004, Day \& Gastel 2006) that provide much more comprehensive guidelines for the complete process of preparing manuscripts from scratch for presentation at conferences and/or submission to peer-reviewed journals. The extensive material in these sources is beyond the scope of this paper, which is much more basically aimed at helping young scientists to construct manuscripts from 'the bottom up' in a concise and cohesive manner.

\section{Formulating an action plan}

The following six steps constitute an 'action plan' that, if put into practice, will hopefully help facilitate quick and effective manuscript preparation (see also Table 1):

1. Writing out a plan.

2. Getting started: writing the descriptive parts of the manuscript.

3. Finishing up: writing the interpretive parts of the manuscript.

4. How to approach the topic.

5. Efficient referencing.

6. Getting the job done.
One of the most important things to remember when writing a manuscript is to try and make the writing as straightforward as possible and to avoid overcomplicating things. I have seen researchers become bogged down in the writing process. This is because they wanted to say too much, believed that the only statistical analyses that mattered were the most complicated ones, or felt that they had to come up with immensely complex explanations for much more straightforward phenomena. The papers I have most enjoyed writing and reading have often been those with the most basic messages. Good science does not necessarily have to be complicated; it just has to describe a well thought-out series of questions and answers (Ford 2009, Underwood 2009). One word of caution here is that there are clearly limits to the extent to which some data and its interpretation can be simplified. It is equally unwise to oversimplify the subject, because this can weaken the impact of the message. The key is to find some level of balance and to go with that. Seeking advice from colleagues or experts in the field can often help in this regard.

It is also important to remember that many of the most interesting studies are 'observational'. The use of a term such as 'experimental' as a synonym for any empirical study designed to answer a question or test a hypothesis has a long history in science, but in the context of this article it is being used in a modern, statistical sense of a 'manipulative experiment'. Likewise, many valuable experiments are not conducted to address a testable hypothesis, but rather to answer an interesting question e.g. how will variation in temperature affect reproduction in a parasitic wasp? These points should be borne in mind when preparing an article for publication.

\section{Getting started: writing up a template for the article and building on it}

Before actually getting started into the 'meat' of the article, it is often wise to draw up a template of the paper, with relevant points of discussion, hypotheses, and appropriate references etc. written down and accessed as necessary. Such a plan can include every section of the paper, starting with the abstract and finishing with the 
summarizing statement in the conclusions. This enables researchers who often have many ideas cluttering their minds about the subject under investigation - like myself - to develop some kind of logical framework with which to build up their paper. One of the things I sometimes do when writing highly detailed papers is to juxtapose the introduction and discussion sections and to put relevant points and information under these headings in point form. This enables me to go back to them when I am writing the paper and to fill in the 'missing' gaps. These gaps can be filled in with full-length paragraphs, if they flow and fit logically with the scientific content. For those of you who prefer a more systematic way of approaching different parts of a paper, it is often best to start with the more basic parts and to leave the interpretive parts until later. This is described in the following sections.

\section{Writing the descriptive parts of the manuscript}

Getting the manuscript started is often the biggest challenge for many scientists: once they get going, then things naturally fall into place. But the question is what to focus on initially? A manuscript may contain many different parts that are all interconnected. Some of the parts are descriptive, some are analytical and some are interpretive. The easiest way to get going is to first write-up the Material and methods section. These generally require little in the way of interpretation, but are merely descriptive, telling the prospective reader (and the referees) what experiments were conducted and how these were set up. I often structure the methods in a logical order e.g. writing-up the methods for the most important experiments that are central to the paper first and lesser experiments later. However, this is subjective. One can also describe the order in which the experiments were conducted,

Table 1. A basic action plan for preparing a manuscript based on manipulative experiments.

Stages of manuscript Details
preparation
preparation

\section{Planning}

2. Descriptive (1)

3. Descriptive (2)

4. Interpretive (1)

5. Interpretive (2)

6. Efficient referencing

7. Final points
- create a framework/template for the article

- put relevant information under different sections in point form

- gather appropriate references and put into a file

- write up Material and methods section

- put in experiments either (a) in the order in which they were conducted, or (b) in order of importance to the study

- write up results, starting with tables and figures, then the statistics (unless these are embedded in the figures)

- ensure the results are placed in the same order as the experiments were described; sub-headings may help

- write up Introduction, providing background to the field of study; this is often the section with heaviest reliance on references

- use last or penultimate paragraph of introduction to explain what was done in the current study - bring in aims and objectives (or hypotheses) at the very end of the introduction

- write up discussion, starting with a brief description of the most important results

- use the following paragraphs to build on information provided in the first paragraph e.g. how do the results compare with those in other studies? What is the significance of the current study in a broader context?

- use the last paragraph to provide conclusions and to explain how the current study can lead to further studies that build on the current one

- the use of bibliographic software

- go over the manuscript thoroughly: are the methods clear enough so that the experiments could be repeated by another lab?

- use spell check to correct mistakes

- let colleagues read the manuscript 
irrespective of their importance in the article. In writing up different experiments, the use of sub-headings is very helpful in this regard. A concise description of the statistical methods used to analyze the data should be placed at the end of this section. Note that the above criteria apply primarily to experiments with few stated objectives. However, if there are more aims and objectives, then these might be kept aside and placed at the end of the introduction once that is prepared (see below). For example, it might make sense to have parallel structures between the two sections. Thus, 'our objectives were (1), (2) and (3); our corresponding methods were ...' and so on.

The Results are easiest to write next. It is often best to produce the tables and figures in the first instance, because these can be discussed with supervisors and colleagues. With them in hand, it is then possible to see whether they need to be revised, and it may also be possible to detect any errors that might be made in statistical analyses. Moreover, using tables and graphs it should also be possible to observe major patterns to be articulated in the results section. Coauthors might wish to look at these before going on to the whole manuscript. Once the data have been analyzed, they can be logically inserted into the paper after the methods section. Remember to ensure that the order in which the results are inserted is the same as were described in the methods section of the manuscript. This makes it easier for the reader to follow the logical progression of the manuscript. Once again, no interpretation is necessary at this stage, but simply a description of what the researcher found in their experiments. One of the most effective ways of writing the results is to present the statistics first and then to very briefly describe what was found. For example, they could be written like: "The relationship between body size and longevity in the parasitoid Venturia canescens was significant (linear regression: $F_{1,35}=6.51, P<0.001$ ). Large parasitoids tended to live up to a week longer than their smaller conspecifics when provided with unlimited food (Fig. 1a)". This way of presenting the results is simple and logical. If desired, terms like 'significant' or 'highly significant' can be excluded from the descriptive statistics. Whereas some researchers think these terms are misleading and convey no information additional to the $P$ value itself, others (like myself) feel that they reinforce the message contained in the $P$ value. For the sake of brevity, statistics can also be embedded in the figures. This would mean that the text would merely say: Large parasitoids provided with unlimited food live significantly longer than smaller conspecifics (Fig. 1a).

\section{Finishing up: writing the interpretive parts of the manuscript}

One of the advantages of writing up the descriptive parts first is that this serves to reinvigorate the researcher's feel for the results and helps in the process of learning to interpret them. This is important because interpreting the results is where the task of writing up experimental results can become most challenging for the inexperienced researcher. First of all, the researcher should possess a solid grounding in the background of their field of study. Without a mature understanding of published studies pertinent to the work at hand, it is very difficult to interpret the broader significance of the results. During the process of setting up and performing the experiments, it is therefore imperative to go through the literature and to highlight studies that may be usefully related to the current study. One note of caution: knowledge of a particular field is not in any way guaranteed by knowing the literature, but being able to interpret it in a meaningful way. Therefore it is often helpful to discuss the field with colleagues or supervisors who may share insights that the writer lacks. The next logical step is to write the Introduction of the paper. The introduction is aimed at providing background to the area(s) in which the research was based, and thus to construct some sort of framework on which to formulate testable hypotheses. Because of this, it is not unusual to find that the introduction contains many more references than any other part of the manuscript.

When starting an introduction, it is useful to make the opening paragraph the broadest in scope and to make guide the reader towards the subject in a more focused way before describing the current study at hand. For example, if 
the subject of the paper was the effects of host size on body size in parasitoid wasps, the author might want to start with something general like: "Body size is considered to be one of the most important traits in animals because it is often strongly correlated with lifetime reproductive success". This statement opens the paper with a very general overview of the importance of body size in many phylogenetically divergent groups of organisms. The next sentence can then be just a little more focused: "One of the greatest challenges in evolutionary biology is to develop an understanding of the factors that affect body size in invertebrates". The author then makes the next sentence even more to the point, e.g. "Parasitoid wasps make excellent subjects for studying constraints on body size evolution in invertebrates because their development is dependent upon the finite resources contained in a single host, whereas insect predators may feed on many prey to attain maturity". Now the topic has been whittled down to the subject of the paper in only three sentences. The writer should cite relevant studies supporting each of the points made above (and possible examples of exceptions where it does not).

After providing a bit more background to the field, the writer eventually needs to bring in what they did in their own research that is relevant to the study of host-size related constraints in parasitoids. This would mean starting a paragraph with something like: "In this study we compared the development of the solitary parasitoid wasp Venturia canescens (Gravenhorst) in different stages of its host, the Indian Meal moth Plodia interpunctella (Hübner)". Some background information about the species under investigation can be provided here (or in the Material and methods if this makes the introduction shorter and 'punchier'). Finally, the author should clearly state the goals of the paper. For example, "The aims of this study are (i) to compare adult parasitoid body mass when developing in different stages (instars of its host), (ii) to compare egg loads in adult female parasitoids of differing adult mass, and (iii) to compare adult longevity in wasps of differing adult mass. We hypothesize that adult parasitoid size is correlated positively with host size and that body size benefits the fitness of female wasps by allowing them to (i) produce more eggs, and/or (ii) to extend their life expectancy". This kind of statement rounds off the introduction and provides reasoning for the background provided earlier. Once the paper is written, the author should come back to the original aims and see if these need to be adjusted or revised. This is because the gist of the paper may have deviated from the 'original script' in the meantime, extra aims may have arisen, or else they do not gel with the stated goals of the journal to which the article will be submitted.

In contrast with the introduction, the Discussion is the part of the manuscript where the author more broadly interprets their own results in the context of what is known in the field. Starting off the discussion can be a difficult task, whereas ideas tend to flow once the writer gets the first paragraph under their belt. There is one really effective way of overcoming 'writer's block' and getting the discussion started. It is essentially quite simple: use the initial paragraph to recite the most important results, and then compare these with other existing studies. Thus, the discussion could start with a phrase like, "The results of this study show that body size in the parasitoid $V$. canescens was strongly affected by the size of its larval host at parasitism. Furthermore, egg load at emergence and adult parasitoid longevity also increased with body size. Other studies have reported that adult size in parasitoids is a function of host size at parasitism". Follow this statement by citing a few (e.g. 3 or 4) published studies that support it. This opens the paper with the most important result of the paper (the relationship between host size and adult parasitoid size) and further provides empirical support for all of the hypotheses that were in the introduction. The findings can then be discussed and compared with those in other published studies on other parasitoid species.

The second and following paragraphs should be used to build on the information provided in the first paragraph, with more details provided revealing how the data here compare with those in other studies and whether they corroborate (or refute) the original hypotheses. The final paragraph of the discussion can be used as a brief summary of the paper (thus, "In this paper we have shown that...") and can also be used 
to suggest ideas for further studies that may or may not be underway or in the planning stages that are relevant to the information presented in the current manuscript. By doing this you are informing your audience that you are involved in further research generated by the ideas in the present study and can save others the time and energy expended in doing so. Alternatively, it can encourage those who would like to work on similar themes to make contact, perhaps setting up the possibility of collaboration.

Finally, here are two other suggestions for writing a good, flowing discussion, which are especially applicable for complex studies. First, when examining and writing up the results, keep notes as to what findings will be especially important to describe and interpret in the discussion. Second, in the case of longer discussions, or in articles with a number of quite divergent questions or hypotheses, use subheadings. When these are clearly presented they provide helpful guides to the reader and allow for emphasis to be given to key findings and ideas.

\section{How to approach the topic}

One of the biggest problems when preparing a manuscript is exactly how to approach the topic of the article, given that there may be different ways of doing so. The important thing to remember is to make sure that the article fits in with the journal to which it will be submitted. It is wholly inappropriate to write an article on the effectiveness of pesticides on the mortality of insect pests in alfalfa fields for strongly evolutionary journals such as Behavioural Ecology and Sociobiology or Evolution, whereas a study on inter-population related polymorphism in the wing color of Monarch butterflies would not fit in with an applied journals such as Biological Control or Journal of Economic Entomology. On the other hand, an article on the factors affecting the development of parasitoid wasps may be approached from both fundamental and applied perspectives, and thus the direction taken is up to the discretion of the writer. Given that many journals are very pressed for space, choosing the appropriate journal can also save considerable time if the article is positively reviewed only for the subject editor to write, "The paper is sound and the reviewers are generally positive but we feel that the material would better fit into another journal". I have had personal experience with this and know the feeling!

\section{Efficient referencing}

As most researchers know, a disproportionate amount of time can be spent assembling references and then typing them into the reference section of the paper. This task is tedious and can take a full day or even longer, depending on the size of the article. In recent years, a number of bibliographic software programs have become available that have made it easy to enter references into manuscripts automatically. These references can be formatted to match the journal to which the manuscript will be submitted. Use of these programs takes a short time to learn and significantly reduces the amount of time spent in this process. It is especially useful in cases where references are entered numerically into the body of the article, as is the case with some of the journals in the Annual Reviews series. Furthermore, using this software guarantees that all references are cited, and should also be used to ensure that all references are in the final version just prior to submission.

\section{Getting the job done}

The art of writing a punchy and interesting manuscript is to focus on the most important details and to avoid the trap of trying to 'say everything' in the fear that important details will be missed. Reviewers generally like to read manuscripts with clear aims and hypotheses (Ford 2009) that were tested with logical and well conducted experiments (Underwood 2009). Moreover, they want to see that the data were correctly analyzed and that the discussion effectively highlights the most interesting details that either corroborate or refute the a priori hypotheses presented in the introduction. Long winded meandering discussions in which the most interesting results are buried amongst jargon are a put-off to most referees and will reduce the chances of the paper 
being accepted, at least in the most rigid journals.

As I alluded earlier, it is important to avoid over-complicating interpretations where a more straightforward explanation would suffice. In some cases this will not be possible, so seek advice from colleagues and others working in the field if you think that guidance is necessary. Many inexperienced scientific writers analyze and interpret their data in the most complex way possible, thus giving the impression that they are 'on top of the subject'. However, this approach can confuse and frustrate reviewers. I maintain that the most enjoyable manuscripts to read and to review are those that have a straightforward message and where the arguments are laid out as clearly as possible. Bear this in mind whenever tackling tricky data sets or masses of conflicting field data.

Once a manuscript is completed, one should always go over the descriptive parts of the paper and ask themselves if someone else could repeat the work, given the descriptions they have provided. It is also important to know if the conclusions are sound and if the paper itself is deemed to be of a quality suitable for publication. For inexperienced researchers, it is always a good exercise to let colleagues and/or a few people working in a similar field read over a final version of the paper. This reduces the risk that something important has been missed, or if the data were wrongly interpreted. With time and practice, having others read over manuscripts becomes less important, but even then it is not necessarily a bad idea, particularly for articles aimed at very rigid journals.

In conclusion, remember that the skill of writing an excellent scientific paper takes time and practice! Do not get discouraged if colleagues or supervisors whittle down your first effort to the bone. My first experience was to write an epic discussion that went on for 10 pages out of the simple fear that I had to say 'everything' about the study. My Ph.D. supervi- sors slashed it to fewer than 3 pages, teaching me a good lesson in prioritizing. With experience, each writer will approach the task in their own way, but it is hoped that some of the general tips described here may nevertheless prove to be helpful. The following points are important to reiterate: write the easiest parts of the paper (generally the descriptive parts) first and the more difficult parts (generally the interpretive parts) once this has been done (see Table 1). Keep the manuscript as simple as possible and try to make it accessible to the reader. Nothing is more frustrating to a reader than having to read and re-read a paper several times to get the gist of it. If these steps are put into practice, the task of writing a paper will become easier and easier, and this may also help the researcher to increase the impact of their written work. Good luck!

\section{Acknowledgements}

I wish to thank Professor John Spence and two anonymous referees for their very helpful comments that have greatly improved the manuscript.

\section{References}

Booth, V. 1993: Communicating in science: writing a scientific paper and speaking at scientific meetings. - Cambridge University Press, U.K.

Day, R. A. \& Gastel, B. 2006: How to write and publish a scientific paper, 6th ed. - Greenwood Press, Connecticut, U.S.A.

Ford, E. D. 2009: The importance of a research data statement and how to develop one. - Annales Zoologici Fennici 46: 82-92.

Gustavii, B. 2003: How to write and illustrate a scientific paper. - Cambridge University Press, U.K.

Malmfors, B., Grossman, M. \& Garnsworthy, P. 2004: Writing and presenting scientific papers. - Nottingham University Press, U.K.

Underwood, A. J. 2009: Components of design in ecological field experiments. - Annales Zoologici Fennici 46: 93-111.

Williams, J. M. 1990: Style: toward clarity and grace. Chicago University Press, Chicago, U.S.A. 